\title{
THE BOCHUM MILKY WAY SURFACE PHOTOMETRY: AN OVERVIEW
}

\author{
Theodor Schmidt-Kaler \\ Astronomical Institute \\ Ruhr-Universität Bochum \\ Postfach 102148, D-4630 Bochum 1 FRG
}

\section{INTRODUCTION}

Photoelectric photometry is inherently much more accurate than photographic work. But large time variations of airglow, inaccurate elimination of bright stars, and the problematic adding-up of different scans may destroy this advantage in the case of surface photometry. On the other hand, the low internal accuracy of photographic photometry and its problematic nonlinearity can be overcome by a sufficient number of plates and by careful photoelectric calibrations. Such measures allow us to take full advantage of the high homogeneity of super-wide-angle photographs over extremely large areas, their global calibration, and their direct imaging. When I started this work, no charge-coupled device or similar device was available. But even today, with such detectors problems would arise for surface photometry in flat-fielding, zeropoints, and bright star elimination. Our camera (Schlosser and Schmidt-Kaler 1977) followed the Henyey-Greenstein design with a f/4.3 Zeiss UV-Sonnar lens (with constant focus from $2000 \AA$ up to $10000 \AA$ ) and $\mathrm{f} / 0.4$ spherical mirror of $30 \mathrm{~cm} \varnothing$, giving an effective $\mathrm{f}=18 \mathrm{~mm}$. The angular resolution, limited by optics and plate, is $4^{\prime}$ in the center, $7^{\prime}$ in the average, and about $20^{\prime}$ at the edges of the field of view $\left(=140^{\circ}\right)$.

\section{THE BOCHUM MILKY WAY ATLAS: QUALITATIVE STUDIES}

Enlargements of 22 representative plates were selected for an atlas covering the whole Milky Way in each of the colors UBVR (Schlosser, Schmidt-Kaler, and Hünecke 1974), with accompanying transparent galactic coordinate-grids. The atlas can be used to study the large-scale structure of the Galaxy. A few examples may be mentioned.

The photographs reveal a complex of dark clouds between $l=90^{\circ}, b=+30^{\circ}$ and $l=210^{\circ}$, $b=-30^{\circ}$ (Schlosser and Görnandt 1984). This Northern Local Dark Cloud System is a coherent system of cellular bubbles of typically $15^{\circ}=30 \mathrm{pc}$ diameter in front of the Local Spiral Arm, inclined by $17^{\circ}$ to the galactic plane, and mirrored in the distribution of young stars, e.g., nebular variables of type I and UV. It is part of the local "shingle" $\mathrm{O}$; similar shingle or corrugation z-structure is well visible for spiral arms $-\mathrm{I}$ and $+\mathrm{I}$.

From red and blue photographs with the galactic center in the middle, we classified our Galaxy as $\mathrm{Sb}\left(-\mathrm{b}^{+}\right)$I-II (Schmidt-Kaler and Schlosser 1973). The zodiacal Gegenschein was resolved into a band structure with three Gaussian components inclined $-2^{\circ} .8,+1^{\circ} .4,+5^{\circ} .7$ against the ecliptic plane (Winkler, Schmidt-Kaler, and Schlosser 1985). 


\section{REDUCTIONS, EXTINCTION, AIRGLOW, ZODIACAL LIGHT, ERRORS}

The geometric transformations from plate to galactic coordinates are better than $\pm 0.1 \mathrm{rms}$. The filter-plate combination was carefully chosen to realize the Johnson UBV system. The additional R magnitude ( $6750 \AA$ ) excludes the influence of interstellar H $\alpha$-regions. In addition, the color equations determined were checked by simultaneous photoelectric measurements (sky scans and stars), which also served to improve the photographic calibration curves and to calibrate them in absolute units. Such measurements also gave the nightly atmospheric extinctions and the airglow data during plate exposures. Using van Rhijn's formula, we found the average height of the airglow emitting layer to be $h=96 \pm 4 \mathrm{~km}$. Dumont's (1965) scattering function was used with airglow parameters after Ashburn (1954), and $f_{A}=1.06$ for light desert soils after Toolin (1965). Finally, the zodiacal light was subtracted, using the model of LevasseurRegourd and Dumont (1980) in V, with solar colors from Tüg and Schmidt-Kaler (1982).

Elimination of bright stars up to $\mathrm{B}=7^{m} .5, \mathrm{~V}=7^{m} .5, \mathrm{U}=8^{m} .5$ was done to $97 \%$ by identification from the CSI catalogue (Ochsenbein et al. 1978). There remained some isolated very bright spots that were identified as stars, and these were removed. The eliminated pixels were replaced by a running mean intensity from the surrounding pixels.

Finally, the photometries of the individual plates (typically $\mathrm{N}=5$ ) were averaged, with geometric accuracy better than \pm 0.1 , and rastered in $0.25 \times 0.25 l, b$ coordinates. Details of reductions are given by Schmidt-Kaler et al. (1983) and by Hoffmann et al. (1989, this volume). Errors of the final surface photometries are typically $\pm 15 \% \mathrm{rms}$ per pixel in $U$ or, more exactly, $4 S_{10} \mathrm{U}$ (additive) and $12 \% I_{u}$ (multiplicative). Maximum systematic errors may be of the same order (or, concerning the additive component, up to $\pm 20 S_{10} \mathrm{U}$ ). The errors in $B$ and $V$ are about half those in $U$, and in R they are about $2 / 3$ of those in $U$.

\section{DARK CLOUDS: QUANTITATIVE STUDIES}

This material can be used for a variety of studies; an excellent example is the study of the Coalsack nebula, where the structure, extinctions, and dust masses were obtained, and correlations to star formation processes were found (Seidensticker and Schmidt-Kaler 1989, this volume). Additional studies are being carried out for isolated large dark clouds $\left(\mathrm{d} \geq 2^{\circ}\right)$ from our data; reliable extinctions can be determined for clouds with $d \geq 0.5$. When these data are combined with star counts from the diffuse galactic light, new information on the albedo and asymmetry factor of the interstellar dust can be obtained.

\section{REFERENCES}

Ashburn, E. V. 1954, J. Atmosph. Terr. Phys., 5, 83.

Dumont, R. 1965, Ann. d'Astrophys. 28, 265.

Hoffmann, B., Kimeswenger, S., Schlosser, W., Schmidt-Kaler, Th., Seidensticker, K. J. 1989, in Proc. IAU 139, Galactic and Extragalactic Background Radiation, ed. S. Bowyer and Ch. Leinert, Dordrecht, Kluwer Academic Pulisher.

Levasseur-Regourd, A. C., Dumont, R. 1980, Astron. Astrophys., 84, 277.

Ochsenbein, F. 1978, Bull. Inf. CDS, No. 15.

Schlosser, W., Schmidt-Kaler, Th., Hünecke, W. 1974, Atlas der Milchstraße, Bochum; see also Vistas in Astr. 21, 447, 1977.

Schlosser, W., Schmidt-Kaler, Th. 1977, Vistas in Astr. 21, 447.

Schlosser, W., Görnandt, V. 1984, Astron. Astrophys. 137, 287.

Schmidt-Kaler, Th., Schlosser, W. 1973, Astron. Astrophys. 29, 409. 
Schmidt-Kaler, Th., Seidensticker, K.J., Pröll, H.J., Schlosser, W., Beck, R. 1983, Astron. Astrophys. Supp. 51, 1.

Seidensticker, K., Schmidt-Kaler, Th. 1989, in Proc. IAU 139, Galactic and Extragalactic Background Radiation, ed. S. Bowyer and Ch. Leinert, Dordrecht, Kluwer Academic Publisher, and references therein.

Toolin, R. B. 1965, in "Handbook of Geophysics and Space Environments," ed. Valley, S. L., Air Force Cambridge Res. Lab., McGraw Hill, New York.

Tüg, H., Schmidt-Kaler, Th. 1982, Astron. Astrophys. 105, 400.

Winkler, Chr., Schmidt-Kaler, Th., Schlosser, W. 1985, Astron. Astrophys. 143, 194.

J. Peebles: Do you accept the usefulness of the exponential disc model, and if so, what is your estimate of the scale length?

Th. Schmidt-Kaler: I accept it but with a proviso: until I get it tested for observations in our Galaxy. Our estimate of the diameter of the main body of the Galaxy tends to be somewhat smaller than that of van der Kruit. How would the Milky Way look without the effect of dust? To answer this we have as a first step corrected for the local dark clouds $(\mathrm{d} \leq 1 \mathrm{kpc})$. These clouds are identified in the catalogue of Feitzinger-Stïwe-Lynds. Using an empirically determined calibration of their opacity classes in Av we get the "Milky Way in U without Dark Clouds". Before we had a very rugged and asymmetric appearance of the Milky Way. Now it appears quite symmetric and rather homogeneous. The symmetry plane is at $b=-0.37$ which means that the Sun is at $Z=+13 \mathrm{pc}$ above the galactic plane.

G. Toller: Pioneer survey analysis indicates that the sun is $12.2 \mathrm{pc}$ above the plane, corroborating Dr. Schmidt-Kaler's statement.

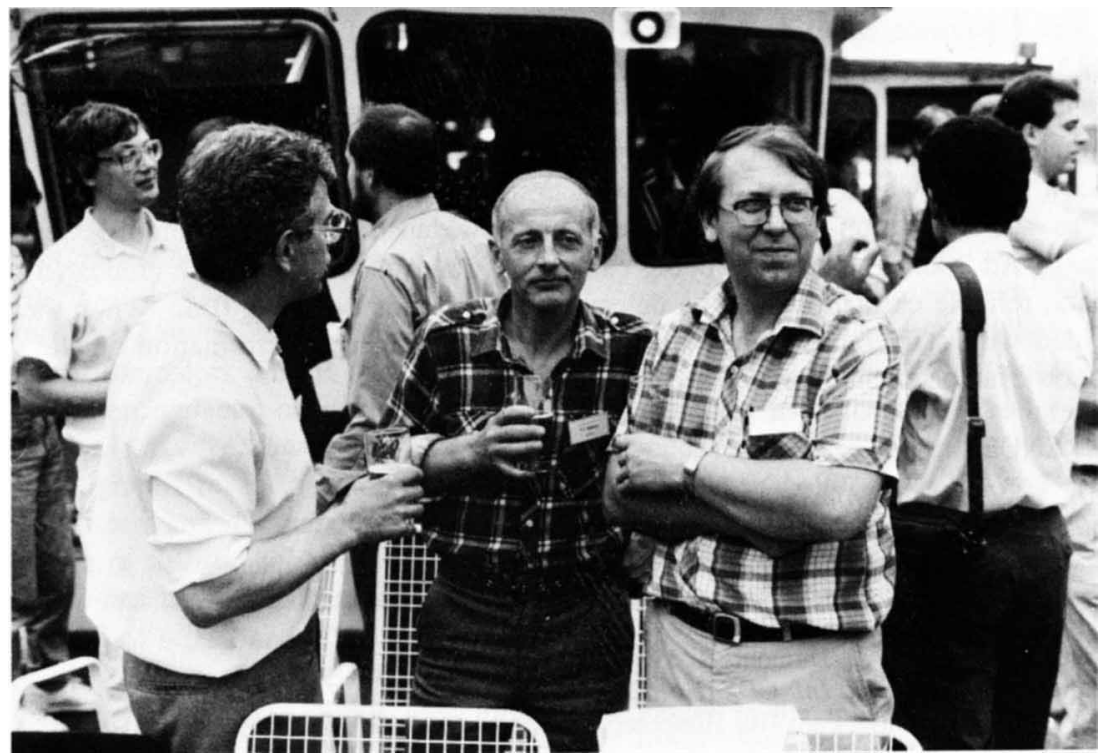

Part of the Soviet delegation:

V. Khersonskii, V. Burdyuzha, and D.A. Skulachev 